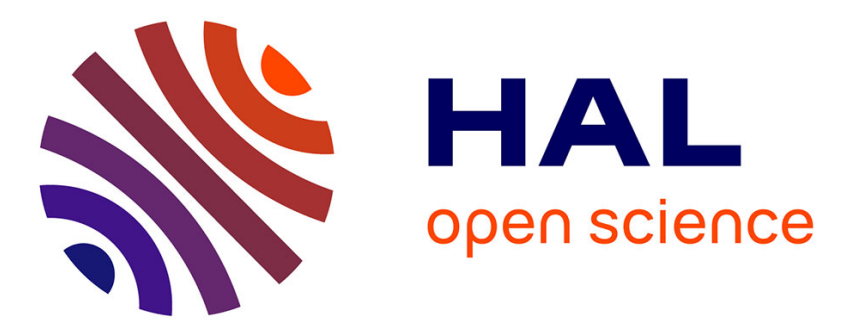

\title{
Efficient generation of third harmonic radiation in air filaments: A revisit
}

Yi Liu, Magali Durand, Aurélien Houard, Benjamin Forestier, Arnaud

Couairon, André Mysyrowicz

\section{- To cite this version:}

Yi Liu, Magali Durand, Aurélien Houard, Benjamin Forestier, Arnaud Couairon, et al.. Efficient generation of third harmonic radiation in air filaments: A revisit. Optics Communications, 2011, 284 (19), pp.4706. 10.1016/j.optcom.2011.05.050 . hal-00852025

\section{HAL Id: hal-00852025}

https://hal-polytechnique.archives-ouvertes.fr/hal-00852025

Submitted on 4 Sep 2013

HAL is a multi-disciplinary open access archive for the deposit and dissemination of scientific research documents, whether they are published or not. The documents may come from teaching and research institutions in France or abroad, or from public or private research centers.
L'archive ouverte pluridisciplinaire HAL, est destinée au dépôt et à la diffusion de documents scientifiques de niveau recherche, publiés ou non, émanant des établissements d'enseignement et de recherche français ou étrangers, des laboratoires publics ou privés. 


\title{
Efficient Generation of Third Harmonic Radiation in Air Filaments: a Revisit
}

\author{
Yi Liu ${ }^{1}$, Magali Durand ${ }^{1}$, Aurélien Houard ${ }^{1}$, Benjamin Forestier ${ }^{1}$, Arnaud Couairon ${ }^{2}$, \\ André Mysyrowicz ${ }^{1, *}$ \\ ${ }^{1}$ Laboratoire d'Optique Appliquée, ENSTA, Ecole Polytechnique, CNRS, \\ Palaiseau, F-91761, France \\ ${ }^{2}$ Centre de Physique Théorique, CNRS, Ecole Polytechnique, F-91128 \\ Palaiseau, France
}

\begin{abstract}
In this paper we revisit the third harmonic generation from a femtosecond laser filament in air and its significant enhancement ( 100 times) with a intercepting pump pulse, which has been reported very recently. The enhanced third harmonic is studied as a function of the pulse polarization, time delay between the pump and signal pulses, laser pulse energy, etc. We provide an explanation for the enhancement of third harmonic based on a quenching of interference effects present near filamentation threshold. Simulations based on a two-color propagation code reproduce well the experimental observations and confirms our interpretation.
\end{abstract}

\section{Introduction}

High-harmonic generation in gases with intense laser pulses has stimulated many research interests because it provides a simple method for achieving laser radiation in UV $\sim$ XUV domain [1-8]. In particular, several papers reported that an efficient conversion $(\sim 0.2 \%)$ from a femtosecond laser pulse to its third harmonic $(\mathrm{TH})$ can be achieved in ambient air or noble gases, where the pulse undergoes filamentation [9-14]. The subject has been pioneered by Aközbek et al. [9]. By including cross phase modulation effects in a two color nonlinear propagation model, these authors showed that the fundamental wave and its third harmonic become quasi phase-locked over the filament length. For a comprehensive review of the filamentation of intense femtosecond laser pulses in air, see Refs. $[15,16]$. Very recently, the subject has been revived by reports that third harmonic generation in air filaments could be increased by 2 orders of magnitude by using a second intense IR pulse (hereafter the pump pulse) to intercept the filament [17-19]. However, the experimental results reported by various groups differ in several aspects. The main discrepancy concerns the effect of time delay $\tau_{d}$ between the filament forming pulse (hereafter the signal pulse) and the intercepting pulse. In Ref. [17] and [19], the authors report a large third harmonic enhancement subsisting for time delays $\tau_{d}$ of several tens of picoseconds. By contrast, in Ref. [18] the authors find that the effect only occurs during the time overlap between pump and filament forming signal pulse. Another discrepancy concerns the influence of the polarizations of the two laser pulses. The authors of references [17, 19] observe an enhancement whether both lasers are parallel or

* Corresponding author : andre.mysyrowicz@ensta-paristech.fr 
orthogonally polarized. In reference [18] only parallel polarized laser pulses can result in TH enhancement. The discrepancies in experimental observations lead naturally to different interpretations of this phenomenon. The authors of Ref. [17] and [19] propose that TH emission is generated inside the local plasma formed at the intersection between the filament and the pump beam. In Ref. [18] the authors suggest that the third harmonic enhancement is due to an increase of the filament length induced by the pump beam.

Meanwhile, Xi and coworkers simulated the propagation of a filament blocked by a water droplet of $40 \mu \mathrm{m}$ on the center and reported an enhancement of the TH [20]. Later, the same group performed experiments by blocking partially the filament core with a thin metallic fiber and confirmed an enhancement of the TH by 1 order of magnitude [21]. Therefore, an immediate question is whether there exists intrinsic relation between these methods for TH enhancement with the one employing a pump filament? Furthermore, is it possible that all the methods share the same physical origin?

In this manuscript, we investigate the $\mathrm{TH}$ enhancement as a function of the incident laser pulse energy, polarization of the pump pulse, and time delay $\tau_{d}$. To interpret our results, we explicitly consider the $\pi$ phase shift which exists between the harmonic wave generated in the front and tail of the filament $[1,22]$. This Gouy phase shift leads to a large cancellation of third harmonic at the end of a short unperturbed filament. By limiting the filament length to its first half distance with a pinhole formed by the filament itself on an aluminum foil, we observe a similar enhancement of $3 \omega$ by two orders of magnitude because the destructive interference is cancelled. At the same time, numerical simulations based on the two color propagation model reproduces well our observations, further confirms our phenomenological interpretation. We also point out that the results of Ref. [17-19] and [21] can be interpreted consistently along the same lines, namely the suppression of destructive interference which prevents back-conversion from the TH to the fundamental pulse.

\section{Experiment results}

In the experiment, we use a chirped pulse amplification (CPA) femtosecond laser system delivering $50 \mathrm{fs}$ laser pulses at $800 \mathrm{~nm}$ with maximum pulse energy of $15 \mathrm{~mJ}$ and a repetition rate of $100 \mathrm{~Hz}$. The pulses have a Gaussian spatial intensity distribution with a diameter of $14 \mathrm{~mm}$ at the output of the CPA system. The output pulse is split into 2 subpulses, the polarization and energy of which can be separately controlled with individual half wave plates and Glan prisms. The two pulses, the pump and filament forming signal pulse, are focused in air with two convex lenses of focal length $f_{1}$ and $f_{2}$ and cross each other under angle $\varphi$. The pump beam focus is positioned to intercept the signal filament at mid distance, as shown in Fig. 1 (a). The time delay between the two pulses at the overlapping point can be varied with a mechanical optical delay line. The far field pattern of the third harmonic generated by the filament is projected on a screen placed $70 \mathrm{~cm}$ beyond the end of the filament, after filtering of the fundamental frequency with UG 11 color filters, as shown in Fig. 1(b). To measure the spectrum and intensity of the TH, the total TH radiation is collected with a convex lens of fused silica with diameter $d=2 \mathrm{~cm}$ and focal distance $f=15 \mathrm{~cm}$ positioned $50 \mathrm{~cm}$ after the end of the filament and sent to a 
spectrometer or a calibrated photodiode after blocking the fundamental frequency with color filters (Fig. 1 (c)).

Fig. 2 (a)-(d) show photographs of the characteristic luminescence track left by the filament plasma string for different pump pulse energies. The corresponding far field patterns of the TH generated by the signal filament are shown in Fig. 2 (e)-(h). The filament length is about $4 \mathrm{~cm}$ for an incident laser pulse energy of $520 \mu \mathrm{J}$ which correspond to $2 P_{\text {cr }}$ where $P_{\text {cr }}=5 \mathrm{GW}$ is the threshold power for filamentation of the 50 fs laser pulse in air. In Fig. 2 (a) and (e), the pump pulse is turned off. In the far field we observe weak residual $800 \mathrm{~nm}$ light and broadband continuum but almost no third harmonic. From (b) to (d), the energy of the pump pulse is 100, 210, $380 \mu \mathrm{J}$, respectively. With the increase of the pump energy, a bright plasma spot appears at the overlapping region and above $E_{\mathrm{p}} \sim 210 \mu \mathrm{J}$ the pump beam starts forming its own filament. At the same time, a significant enhancement of third harmonic is observed in the far field. At low pump pulse energy, below $E_{\mathrm{p}}=100 \mu \mathrm{J}$, a centro-symmetrical circular emission pattern of the TH centered on the filament axis is observed. At higher pump pulse energy $\left(E_{\mathrm{p}}>210 \mu \mathrm{J}\right)$, part of the $\mathrm{TH}$ emission appears at a larger angle, preferentially in the direction orthogonal to the plane formed by the two crossing filaments. Figure 3 shows the spectrum of the third harmonic with and without the pump pulse, corresponding to Fig. 2(a) and (d). An enhancement of the third harmonic energy by a factor 100 is observed for a pump energy of $380 \mu \mathrm{J}$.

We performed systematic measurements to address the role of the pump pulse polarization and the time delay $\tau_{d}$ between the two pulses in the enhancement of the third harmonic. Figure 4 shows the enhanced TH signal as a function of the time delay $\tau_{d}$ for different energies and polarizations of the pump pulse. For $E_{p}=120 \mu \mathrm{J}$, an enhanced TH signal is observed in the case of parallel polarization within a delay of a few hundred femtoseconds (Fig. 4(a)). These observations are similar to those in Ref. [18]. At high pump pulse energy of $520 \mu \mathrm{J}$ (Fig. 4 (d)), almost no difference is found between the two laser polarizations, in agreement with Refs. [17] and [19]. For pump energy between 120 $\mu \mathrm{J}$ and $520 \mu \mathrm{J}$, an intermediate behavior is observed, in which the two polarizations produce different third harmonic signals during the $\sim 200$ fs time overlap, while after no difference is observed (Fig. 4 (b) and (c)). We further measured the maximum enhanced third harmonic as a function of the pump pulse energy systematically for the two orthogonal pump polarizations. The results are presented in Fig. 5. Consistent with the above results, for pump pulse energy relatively low $(<500 \mu \mathrm{J})$ the two polarizations exhibit significant difference while a similar enhancement is observed for higher energy pump pulse.

We further compared the TH signal from a free propagating filament with the maximum enhanced TH signal in the presence of a pump filament with $E_{\mathrm{p}}=300 \mu \mathrm{J}$. Results are presented in Fig. 6 (a). For low energy pulse $(<400 \mu \mathrm{J})$ an enhancement by a factor of 100 is observed, while this improvement effect weakens gradually with the increment of the incident energy. 
Concerning the underlying mechanism for the TH enhancemet, we find some clues in Fig. 2. In this figure it is seen that the filament luminescence track is gradually shortened in the presence of the pump pulse. Yet the maximum $\mathrm{TH}$ radiation is obtained when the filament is the shortest. This observation suggests that the $\mathrm{TH}$ generated in the first part of the filament is largely cancelled by the second half of the filament in the far field.

To check this hypothesis, we employed another method to reduce the length of the filament, leaving its first part unperturbed. We inserted a $150 \mu \mathrm{m}$ thick aluminum foil in the middle of an intense $4 \mathrm{~cm}$ long filament formed by a $600 \mu \mathrm{J}$ pulse focused with lens of $f=1 \mathrm{~m}$. After about 2 seconds, the intense filament core starts to drill a hole on the metallic foil and partially penetrates through it. As a result, a small diaphragm with a diameter corresponding to the intense filament core is realized. We monitored the $\mathrm{TH}$ signal in the far field during this process and present the results in Fig. 7 as a function of the exposition time. The evolution of the diaphragm is also presented as inset. It is not surprising to find that the diaphragm enlarges gradually, followed by a rather stable state with constant diameter with the accumulation of laser shots. However, the TH energy presents a maximum around $t=2 \sim 3 \mathrm{~s}$ and decreases substantially after 4 seconds when a larger opening is achieved. We argue that with a smaller pinhole of around $60 \mu \mathrm{m}$, corresponding to the exposition time from $2 \mathrm{~s}$ to $4 \mathrm{~s}$, the laser energy reservoir surrounding the filament core is largely blocked, preventing further filamentation after the pinhole $[23,24]$. Meanwhile, the third harmonic wave generated inside the intense core of the filament before the diaphragm is transmitted through the pinhole. With the further enlargement of the pinhole opening, the transmitted laser power becomes larger than the $P_{\text {cr. }}$. As a result, a hypogenetic filament develops after the pinhole $[23,24]$ and serves as additional $\mathrm{TH}$ source, contributing to the total $\mathrm{TH}$ yield in the far field. Therefore, the observed decreasing of the TH after $t=3 \mathrm{~s}$ in Fig. 7 lead to the speculation that the TH generated after the pinhole contribute oppositely to the total $\mathrm{TH}$ yield, comparing to the $\mathrm{TH}$ produced before the diaphragm.

To monitor the amount of $\mathrm{TH}$ generated in different sections of the filament, we displaced the Al foil along the filament axis and recorded the corresponding maximum $\mathrm{TH}$ yield. The results are presented in Fig. 8. The TH signal increases in the first section of the filament, reaches a maximum and then decreases in the second half section. This observation supports the hypothesis that the $\mathrm{TH}$ radiation generated in the filament tail interferes destructively with the TH generated in the first section. It is further confirmed by the following measurement. The filament is first intercepted at mid distance by the pump pulse. A pre-formed $100 \mu \mathrm{m}$ diaphragm is then placed beyond the crossing point and the TH is measured again by displacing the diaphragm along increasing $z$. The result is also shown in Fig. 8. As can be seen, the TH signal keeps almost constant after the crossing point, instead of decreasing rapidly as it is the case with a fully developed filament. This observation suggests that the dense plasma at the crossing point actually acts as a blocker that partially stops the filament formation and hence arrests the back conversion of the $\mathrm{TH}$ generated in the upstream half filament into the fundamental frequency. 
We therefore conclude that the $\mathrm{TH}$ produced on the leading and trailing parts of the filament possess opposite carrier phase, which is at the origin of the large cancellation of $\mathrm{TH}$ at the end of a fully developed filament. In the presence of an intercepting pump filament or other objects (small size pinhole, thin copper wire, water droplet, etc), the development of the filament is suppressed after the obstacle, breaking the large cancellation of the $\mathrm{TH}$ at the end of the filament. As a result, an enhancement of the total TH yield is generally observed.

Finally, we compared the maximum enhanced $\mathrm{TH}$ as a function of the input energy in the presence of the Al diaphragm with that of an unperturbed filament, as shown in Fig. 6 (b). A basically similar scenario is found comparing to Fig. 6(a), confirming the same physical mechanism underlying the two methods for $\mathrm{TH}$ enhancement.

\section{Numerical simulations and discussion}

To understand the $\mathrm{TH}$ development inside the filament, we performed simulations based on an extension of the model developed by Aközbek et al. [9]. The standard modeling of filamentation by a nonlinear envelope equation of the nonlinear Schrödinger type for the laser field coupled with an evolution equation for the electron density in the ionizing medium traditionally relies on the slowly varying wave approximation [15]. An associated constraint requires that the pulse be longer than a few cycles for the fundamental frequency. It is now standard to relax this constraint by properly describing space-time focusing and self-steepening of the pulse in the framework of the slowly evolving wave approximation (SEWA) [25]. Our extension of the Aközbek et al. model consists in applying the SEWA to the full vectorial model for the coupled system of propagation equations related to the fundamental pulse and its third harmonic. See Ref. 26 for a similar derivation. The electric field is therefore written in terms of the fundamental $E_{\omega_{0}}(\vec{r}, t, z)$ and third harmonic $E_{3 \omega_{0}}(\vec{r}, t, z)$ envelopes:

$$
E=E_{\omega_{0}} \exp \left\lfloor i\left(k_{\omega_{0}} z-\omega_{0} t\right)\right\rfloor+E_{3 \omega_{0}} \exp \left\lfloor i\left(k_{3 \omega_{0}} z+\Delta k z-3 \omega_{0} t\right)\right\rfloor
$$

with $k_{\omega_{0}} \equiv k\left(\omega_{0}\right), k_{3 \omega_{0}} \equiv k\left(3 \omega_{0}\right)$, and $\Delta k=3 k\left(\omega_{0}\right)-k\left(3 \omega_{0}\right)=-5 \mathrm{~cm}^{-1}$ (air at $800 \mathrm{~nm}$ ) where $k(\omega)$ denotes the dispersion relation in air [27]. The propagation equations for the fundamental and third harmonic pulses are written in the Fourier domain for $\hat{E}_{\omega_{0}}(\vec{r}, \omega, z)$ and $E_{3 \omega_{0}}(\vec{r}, \omega, z)$ :

$$
\begin{aligned}
& 2 i K_{\omega_{0}}(\omega) \frac{\partial \hat{E}_{\omega_{0}}}{\partial z}=-\left[\Delta_{\perp}+D_{\omega_{0}}(\omega)\right] \hat{E}_{\omega_{0}}+i \frac{\omega^{2}}{c^{2}} \hat{N}_{K, \omega_{0}}-i k_{\omega_{0}} \hat{N}_{P, \omega_{0}}-\frac{\omega}{c} \hat{N}_{M, \omega_{0}} \\
& 2 i K_{3 \omega_{0}}(\omega)\left(\frac{\partial \hat{E}_{3 \omega_{0}}}{\partial z}-\Delta k \hat{E}_{3 \omega_{0}}\right)=-\left[\Delta_{\perp}+D_{3 \omega_{0}}(\omega)\right] \hat{E}_{3 \omega_{0}}+i \frac{\omega^{2}}{c^{2}} \hat{N}_{K, 3 \omega_{0}}-i k_{3 \omega_{0}} \hat{N}_{P, 3 \omega_{0}}-\frac{\omega}{c} \hat{N}_{M, 3 \omega_{0}}
\end{aligned}
$$

Here the dispersive terms for each component $D_{\omega_{0}} \equiv k^{2}(\omega)-K_{\omega_{0}}^{2}(\omega)$ and $D_{3 \omega_{0}} \equiv k^{2}(\omega)-K_{3 \omega_{0}}^{2}(\omega)$, where $\quad K_{\omega_{0}} \equiv k\left(\omega_{0}\right)+k_{0}^{\prime}\left(\omega-\omega_{0}\right) \quad, \quad k_{0}^{\prime} \equiv d k /\left.d \omega\right|_{\omega_{0}}$ and $K_{3 \omega_{0}}=k\left(3 \omega_{0}\right)+k_{0}^{\prime}\left(\omega-3 \omega_{0}\right)$, are not truncated to a specific order and the frequency dependent operators in equations (2) and (3) make the model valid for pulses as short as a single cycle. Note that group velocity mismatch is included in the dispersive terms for the 
third harmonic pulse since both the fundamental and the third harmonic pulses are propagated in the frame of the fundamental pulse $\tau=t-k_{0}^{\prime} z$, where $t$ denotes time in the laboratory frame. As emphasized by Kolesik et al., because of decomposition (1), the model assumes that the spectrum of the fundamental pulse does not overlap that of the third harmonic [11]. This assumption is well satisfied in our calculations. The nonlinear terms in Eqs. (2) and (3) describe (i) the optical Kerr effect with self- and cross-phase modulation, third-harmonic generation and back-conversion:

$$
\begin{aligned}
& N_{K, \omega_{0}}=2 i n_{\omega_{0}} n_{2}\left[\left(\left|E_{\omega_{0}}\right|^{2}+2\left|E_{3 \omega_{0}}\right|^{2}\right) E_{\omega_{0}}+E_{\omega_{0}}^{* 2} E_{3 \omega_{0}}\right] \\
& N_{K, 3 \omega_{0}}=2 i n_{3 \omega_{0}} n_{2}\left[\left(\left|E_{3 \omega_{0}}\right|^{2}+2\left|E_{\omega_{0}}\right|^{2}\right) E_{3 \omega_{0}}+E_{\omega_{0}}^{3} / 3\right]
\end{aligned}
$$

(ii) plasma absorption and plasma defocusing for the fundamental and the third harmonic pulses:

$$
\begin{aligned}
& N_{P, \omega_{0}}=\sigma_{\omega_{0}}\left(1+i \omega_{0} \tau_{c}\right) \rho E_{\omega_{0}} \\
& N_{P, 3 \omega_{0}}=\sigma_{3 \omega_{0}}\left(1+i 3 \omega_{0} \tau_{c}\right) \rho E_{3 \omega_{0}}
\end{aligned}
$$

(iii) nonlinear (multiphoton) absorption for both components:

$$
\begin{aligned}
& N_{M, \omega_{0}}=n_{\omega_{0}} \beta_{K_{\omega_{0}}}\left|E_{\omega_{0}}\right|^{2 K_{\omega_{0}}-2} E_{\omega_{0}} \\
& N_{M, 3 \omega_{0}}=n_{3 \omega_{0}} \beta_{K_{3 \omega_{0}}}\left|E_{3 \omega_{0}}\right|^{2 K_{3 \omega_{0}}-2} E_{3 \omega_{0}}
\end{aligned}
$$

The various parameters in Eqs. (4-6) are: the linear refractive index $n_{\omega_{0}}$ and $n_{3 \omega_{0}}$, the cross sections for inverse Bremsstrahlung $\sigma_{\omega_{0}}=5.5 \times 10^{-20} \mathrm{~cm}^{2}$ and $\sigma_{3 \omega_{0}}=6.1 \times 10^{-21} \mathrm{~cm}^{2}$, the cross sections for multiphoton absorption $\beta_{K_{\omega_{0}}}=1.8 \times 10^{-94} \mathrm{~cm}^{13} \mathrm{~W}^{-7}$ and $\beta_{K_{3 \omega_{0}}}=1.4 \times 10^{-26} \mathrm{~cm}^{3} \mathrm{~W}^{-2}$, the numbers of photons $K_{\omega_{0}}=8$ and $K_{3 \omega_{0}}=3$ involved in the process at the fundamental and third harmonic frequencies, respectively; the collision time $\tau_{c}=350 \mathrm{fs}$ in air and the nonlinear index coefficient $n_{2}$. We performed simulations for 2 values of the nonlinear index coefficient: $n_{2}=3.2 \times 10^{-19} \mathrm{~cm}^{2} / \mathrm{W}$ and $n_{2}=1.8 \times 10^{-}$ ${ }^{19} \mathrm{~cm}^{2} / \mathrm{W}$. The first value is more appropriate for pulse durations of $50 \mathrm{fs}$ or more while the second value corresponds to pulse durations for which the role of the retarded Kerr effect is negligible.

Ionization of air is finally described by the evolution equation for the electron density $\rho$ with source terms corresponding to multiphoton ionization and avalanche processes

$$
\frac{\partial \rho}{\partial t}=\left(\sigma_{K_{\omega_{0}}}\left|E_{\omega_{0}}\right|^{2 K_{\omega_{0}}}+\sigma_{K_{3 \omega_{0}}}\left|E_{3 \omega_{0}}\right|^{2 K_{3 \omega_{0}}}\right)\left(\rho_{n t}-\rho\right)+\left(\sigma_{\omega_{0}}\left|E_{\omega_{0}}\right|^{2}+\sigma_{3 \omega_{0}}\left|E_{3 \omega_{0}}\right|^{2}\right) \frac{\rho}{U_{i}}
$$

where $\sigma_{K_{\omega_{0}}}=3.7 \times 10^{-96} \mathrm{~cm}^{16} \mathrm{~W}^{-8} \mathrm{~s}^{-1}$ and $\sigma_{K_{3 \omega_{0}}}=2.5 \times 10^{-28} \mathrm{~cm}^{6} \mathrm{~W}^{-3} \mathrm{~s}^{-1}$ denote the cross sections for multiphoton ionization at the fundamental and third harmonic frequencies, $\rho_{n t}$ denotes the density of neutral oxygen molecules which is the main ionized species at intensities of a few $10^{13} \mathrm{~W} / \mathrm{cm}^{2}$ and $U_{\mathrm{i}}$ denotesits ionization potential. 
In Fig. 9, we present the evolution of the fundamental and $\mathrm{TH}$ for an incident pulse of low energy $(1 \mathrm{pJ})$. We follow simultaneously the maximum intensity of the fundamental and third harmonic pulses, their Full Width at Half Maximum (FWHM) calculated from their fluence distribution, and the energy contained in a cylinder of radius 100 microns which mimics the experimental diagnostic. The propagation of the fundamental pulse is governed by the laws of Gaussian optics. It is seen that the $\mathrm{TH}$ energy increases progressively before the focus and decreases again after the focus. The oscillation period of the TH pulse due to the phase mismatch is clearly visible. The amplitude of these oscillation decreases when the focusing geometry is tighter as in our experiments compared to simulations in the following. In the far-field, all the TH energy returned to the pump. This behavior has been mainly attributed to the Gouy phase shift between the fundamental and third harmonic [1, 9, 22], which leads to destructive interference between the third harmonic waves generated before and after the focus.

In Fig. 10, we present the same quantities but inside a short filament, calculated for parameters corresponding to our experiments (waist at the focus of $30 \mu \mathrm{m}$ ) and pulse energies of 100 and $500 \mu \mathrm{J}$. The fundamental pulse forms a standard short filament with increasing length when the input energy increases. The propagation of the TH pulse exhibits attenuated contrast in the oscillations. For both pulses with input energy of 100 $\mu \mathrm{J}$ and $500 \mu \mathrm{J}$, it is seen that the $\mathrm{TH}$ decreases slightly after its maximum but its energy is not entirely given back to the fundamental pulse. Although the energy of the TH pulse at the end of filamentation may be several decades below the maximum energy reached in the filament, it retains an energy of several nanojoules for an input pulse of $500 \mu \mathrm{J}$. The beam width of the third harmonic presents discontinuities in the nonlinear regime. This is due to the fact that the TH beam exhibits an axial and a conical component as will be illustrated below. Only the axial component gives its energy back to the pump whereas the conical component stores most of the energy retrieved at the end of the filamentation stage.

In Fig. 6 (c, d), we presented the $\mathrm{TH}$ yield from a fully developed filament for fundamental pulse energies up to $2.2 \mathrm{~mJ}$. To mimic the function of the Al diaphragm, we simulate it with a numerical aperture with a diameter of $100 \mu \mathrm{m}$ and monitor the maximum $\mathrm{TH}$ contained in this area during filamentation. The results are shown in Fig. 6(c) for a nonlinear index coefficient $n_{2}=3.2 \times 10^{-19} \mathrm{~cm}^{2} / \mathrm{W}$ corresponding to the critical power of $3 \mathrm{GW}$, and in Fig. 6(d) for $n_{2}=1.8 \times 10^{-19} \mathrm{~cm}^{2} / \mathrm{W}$ corresponding to the critical power of $5 \mathrm{GW}$. The qualitative agreement between the experiment and simulation confirms our above phenomenological explanation. The energy of the TH pulse increases rapidly for increasing but low energies of the fundamental pulse. Above $500 \mu \mathrm{J}$, the $\mathrm{TH}$ energy still increases but at a slower rate. We note that the difference between the $\mathrm{TH}$ energy passing through the aperture of $100 \mu \mathrm{m}$ and the total TH energy calculated at the end of the filament decreases at large fundamental pulse energies. This is due to the fact that increasing nonlinearity due to the longer filamentation length quenches the destructive interference responsible for the back conversion of the energy in the axial component of the TH pulse. Consequently, the fraction of TH energy that remains in the axial component is closer to the maximum $\mathrm{TH}$ energy for large fundamental pulse energies. 
Fig. 11 presents the space-time dynamics of the fundamental (first column) and $\mathrm{TH}$ (second column) pulses as functions of the propagation distance around the linear focus for an incident energy of $500 \mu \mathrm{J}$. The fundamental pulse follows a standard filamentation dynamics starting with self-focusing, followed by the generation of a plasma that defocuses the trailing part (positive times) of the pulse and forms a ring-shaped beam surrounding the intense central part; a refocusing process finally brings part of the energy of this ring back to the center but the fluence profile at the end of the filament still exhibits an intense axial beam surrounded by a conical beam. The dynamics of the TH pulse partly follows that of the fundamental since it is the third harmonic generation term (last term in Eq. (4b)) which plays the most important role among nonlinearities affecting the TH pulse. Therefore, we observe the formation of a ring in the TH pulse which is also clear from the time integrated (fluence) profiles plotted as white curves on each figure. As previously shown $[9,12,14]$, this TH ring is not only the result of third harmonic generation by the ring-shaped fundamental pulse but also the result of a phase matching process involving the axial part of the fundamental pulse and a preferential angle for the $\mathrm{TH}$ pulse. The structures which are not visible in the fundamental pulse but appear in the $\mathrm{TH}$ pulse profile indicate the latter phase matching process leading to conical $\mathrm{TH}$ generation. It is clear that the conical part of the TH pulse do not spatiotemporally overlap with the conical part of the fundamental pulse. This confirms that the energy corresponding to the conical emission of the TH pulse cannot go back to the pump as four wave mixing governed by the last term in Eq. (4a), which should induce this backconversion, is prevented in the absence of spatial and temporal overlap. In contrast the axial part of the fundamental and TH pulses do overlap over a significant propagation distance within the filament, thereby allowing for back conversion from the TH pulse to the fundamental.

As a final comment, we would like to come back to the discrepancies concerning the $\mathrm{TH}$ enhancement with a pump filament and the comparison of all these methods. In our opinion, previous experiments reporting strong TH enhancement with short perturbed filaments can be interpreted consistently along the same lines. The apparent contradictory results concerning the delay can be reconciled by considering the type of perturbation introduced in the filament. For low power pump pulse, like the results in Ref. [18] and ours in Fig. 4 (a), an enhanced TH signal is observed in the case of parallel polarization and within a delay of a few hundred femtoseconds. This can be attributed to the fact that a plasma blocker is formed by the interference pattern between the pump and signal laser fields, acting as an efficient interference grating. This requires that the signal and pump pulses have the same polarization. At higher pump pulse energies, almost no difference is found between the two laser polarizations [17, 19]. This is similar to the results presented in Fig. 4 (d). This phenomenon is due to the fact that the pump intensity is now much higher than that of the signal pulse and the plasma formed by the pump pulse acts now as a filament blocker. The polarization of the pump pulse is no longer crucial for the $\mathrm{TH}$ enhancement since the contribution of the relatively weaker signal pulse to the formation of the plasma blocker is negligible. Concerning the other methods for $\mathrm{TH}$ enhancement, blocking partially the filament core with water droplet or thin metallic fiber, we argue that the roles of these obstacles are also to perturb the filament develop and lead 
to break of the large cancellation of TH generated inside, like it was pointed out by the authors $[20,21]$.

\section{Conclusion}

We studied the enhanced third harmonic generation from a femtosecond laser filament intercepted by a pump femtosecond pulse. Systematic measurement of the enhanced TH as function of experimental parameters, such as the pulse polarization, time delay and pulse energies, have been performed. Based on these results, we concluded that the enhancement of the $\mathrm{TH}$ originates from the breaking of the large cancellation of the $\mathrm{TH}$ in a filament perturbed by a pump pulse. Numerical simulations have been performed to examine the dynamics of the $\mathrm{TH}$ generation in a filament. It is revealed that for pulse with relatively low energy pulse, it is the geometrical Gouy phase shift that is responsible for the TH cancellation inside the filament. Meanwhile, for more energetic pulses, the nonlinear propagation effects overcome largely the effect of the Gouy phase shift so that substantial TH survives at the end of a free propagating filament, as confirmed by the experiments.

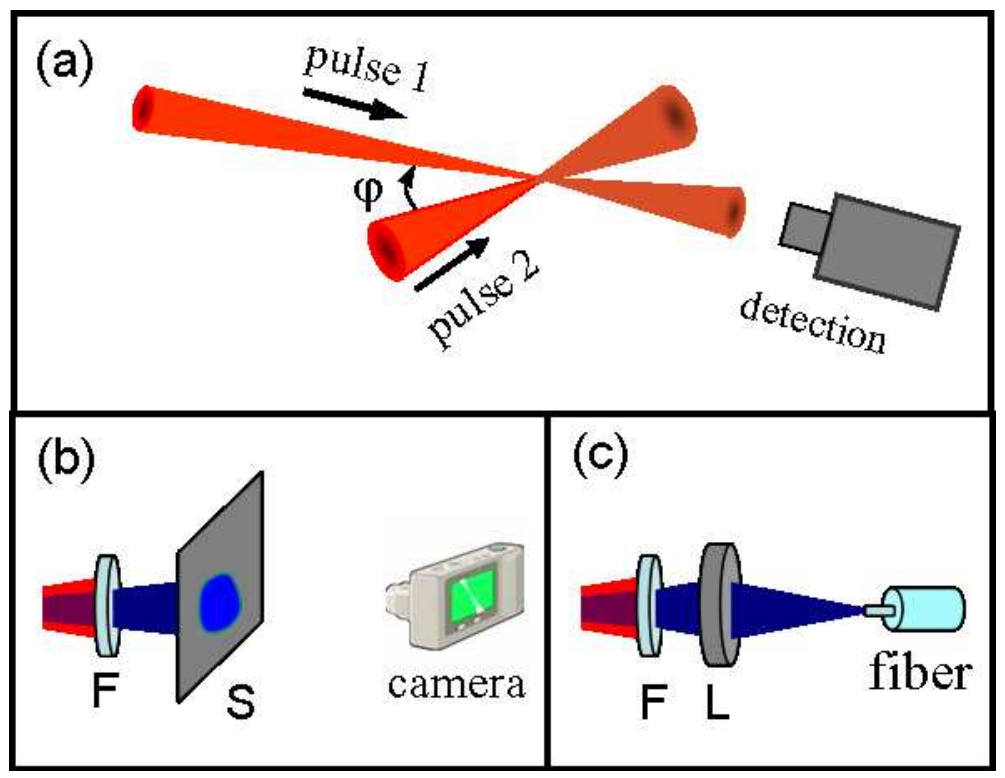

Fig. 1. Schematic experimental setup. (a) Two femtosecond pulses form filaments in air, which cross with angle $\varphi$. The detection is detailed in (b) and (c). (b) the pulse 1 after interaction is filtered by color filters (F) and projected on a paper screen $\mathrm{S}$. The fluorescence induced by the third harmonic on the screen is taken by a camera. (c) The third harmonic after the filters is focused by a fused silica lens (L) and detected by a fibre spectrometer or calibrated photodiode. 

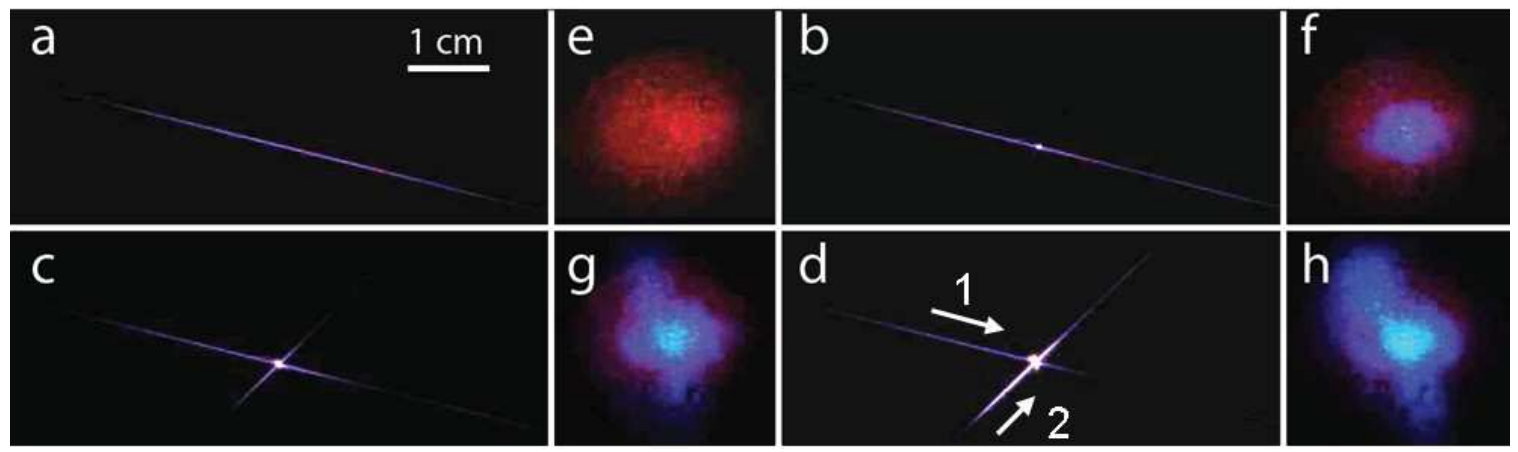

Fig. 2. Photos of the characteristic plasma luminescence of a free propagating filament (a) and filaments intercepted by a pump pulse (b)-(d). The signal pulse (1) and pump pulse (2) are focused by convex lenses of $f_{1}=100 \mathrm{~cm}$ and $f_{2}=50 \mathrm{~cm}$. The arrows in (d) represent their propagation directions. The pump pulse energy are $100,210,380 \mu \mathrm{J}$ for (b)-(d). (e)-(h), the images of the third harmonic generated by the signal filament and the residual IR component corresponding to (a)-(d).

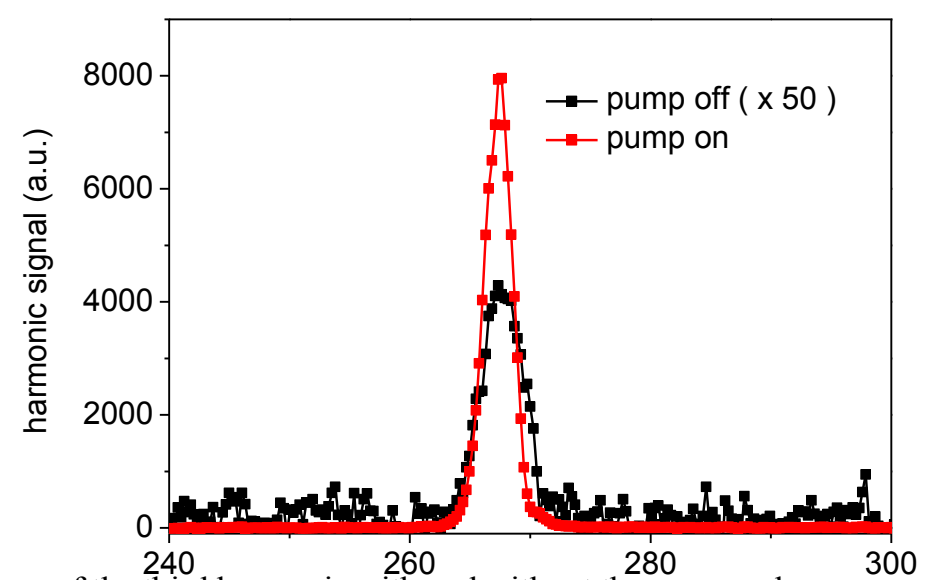

Fig. 3. Spectrum of the third harmonic with andereithout (the pump pulse, corresponding to the case of Fig. 2(a) and (d). 

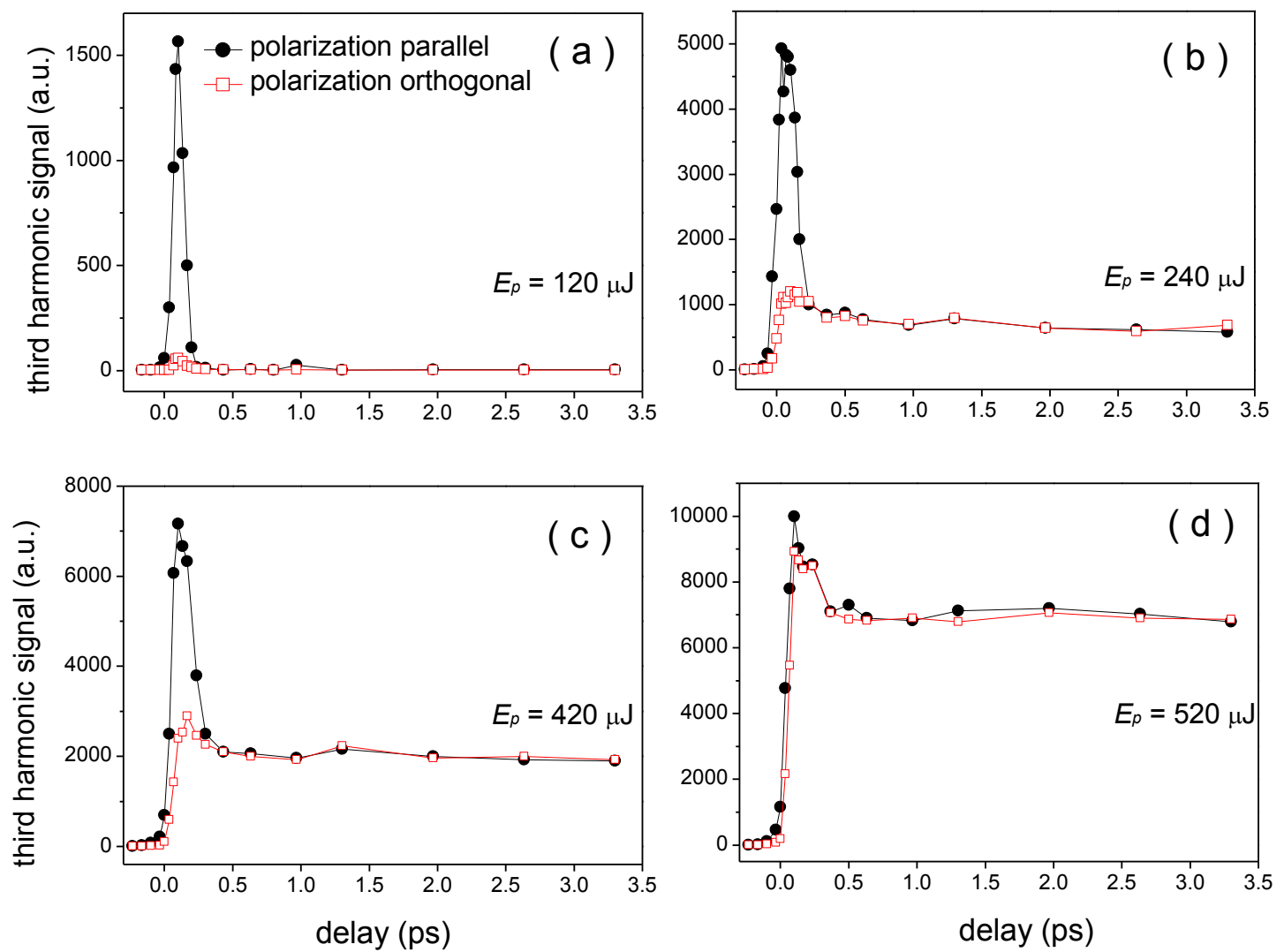

Fig. 4. Third harmonic signal as a function of delay $\tau_{d}$ for different pump polarization and pump pulse energy. The lenses used to focus the signal and pump pulse are $f_{1}=100 \mathrm{~cm}$ and $f_{2}=15 \mathrm{~cm}$. The cross angle $\varphi$ of the two filaments is $90^{\circ}$. The energy of the signal pulse is $320 \mu \mathrm{J}$. 


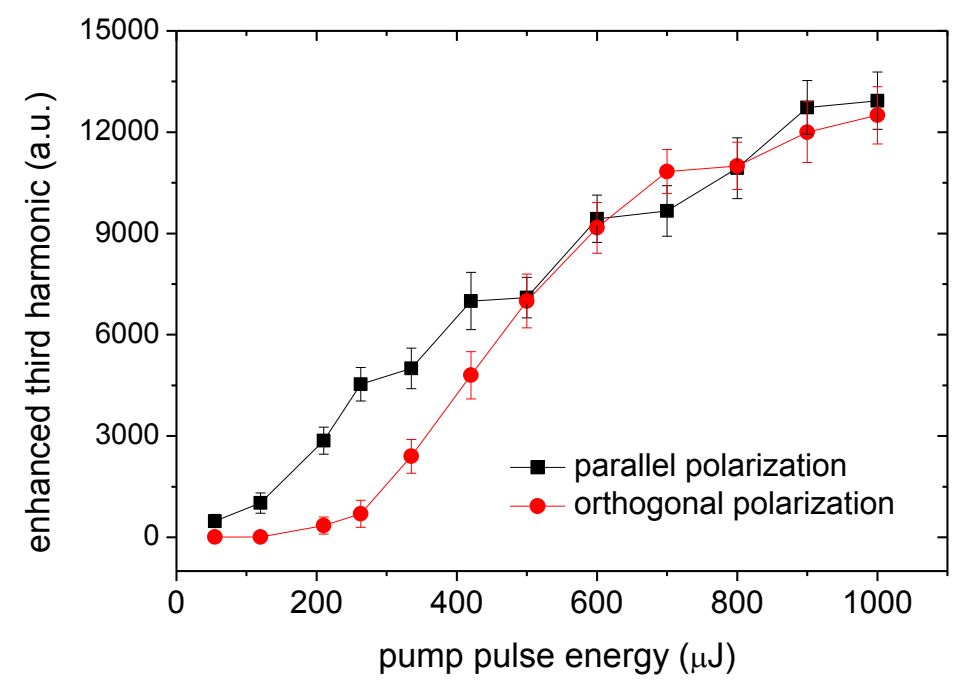

Fig. 5. The enhanced TH signal as a function of the pump pulse energy, while the signal filament pulse energy is fixed to $330 \mu \mathrm{J}$. The experiment conditions are the same as in Fig. 4.
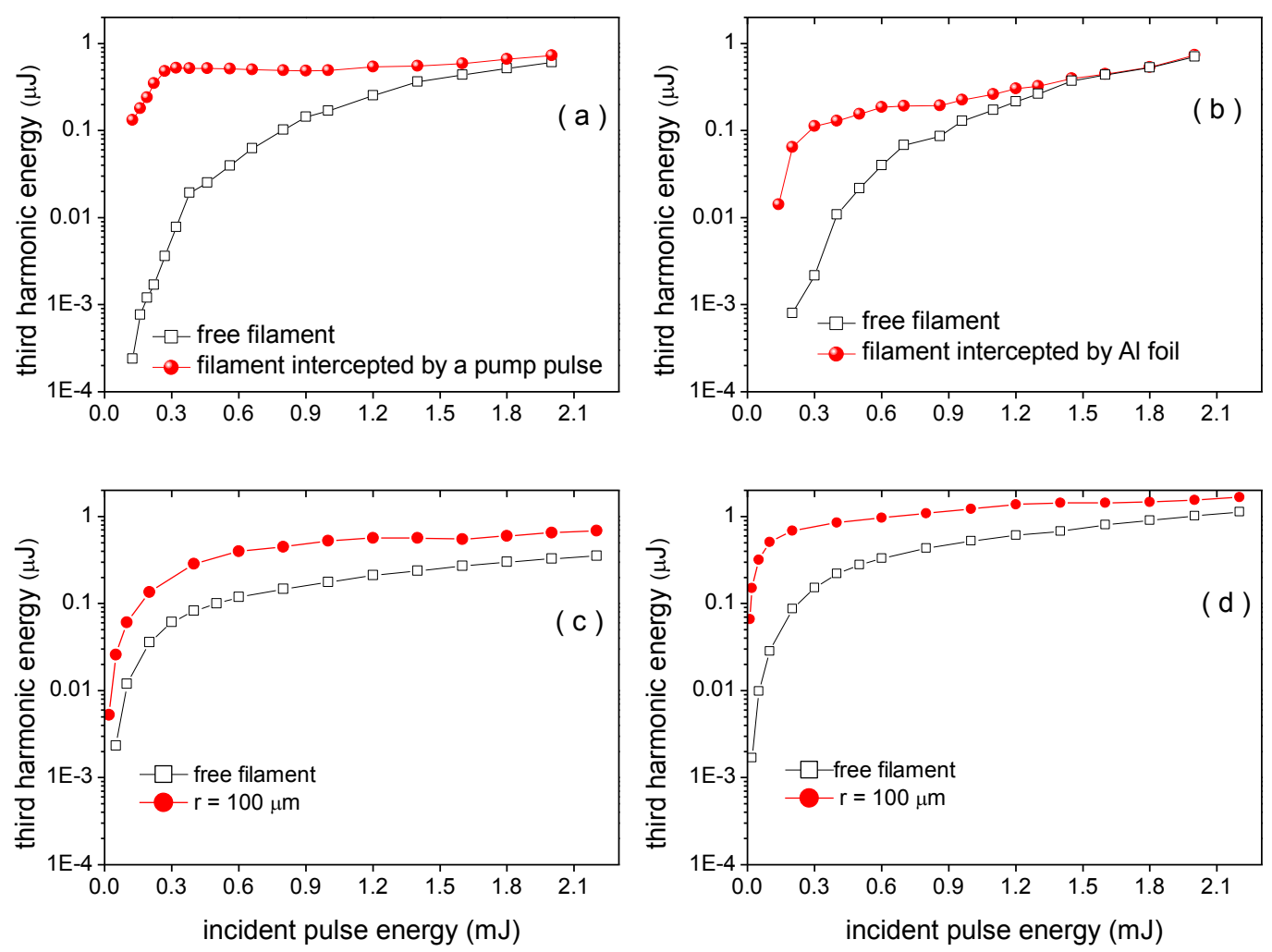

Fig. 6. (a) The measured TH signal from a free propagating filament as a function of the signal pulse energy, and the maximum TH signal from filament intercepted by a $300 \mu \mathrm{J}$ pump pulse. (b) Same as in (a) but the filament is intercepted by an $\mathrm{Al}$ foil. (c) Calculated TH energy from a free filament $(10 \mathrm{~cm}$ after the focus) and the maximum TH energy contained in a $100 \mu \mathrm{m}$ aperture as functions of the input energy of the 
fundamental pulse. The nonlinear index coefficient is $n_{2}=3.2 \times 10^{-19} \mathrm{~cm}^{2} / \mathrm{W}$. (d) Same as in (c) but the nonlinear index coefficient is $n_{2}=1.8 \times 10^{-19} \mathrm{~cm}^{2} / \mathrm{W}$.

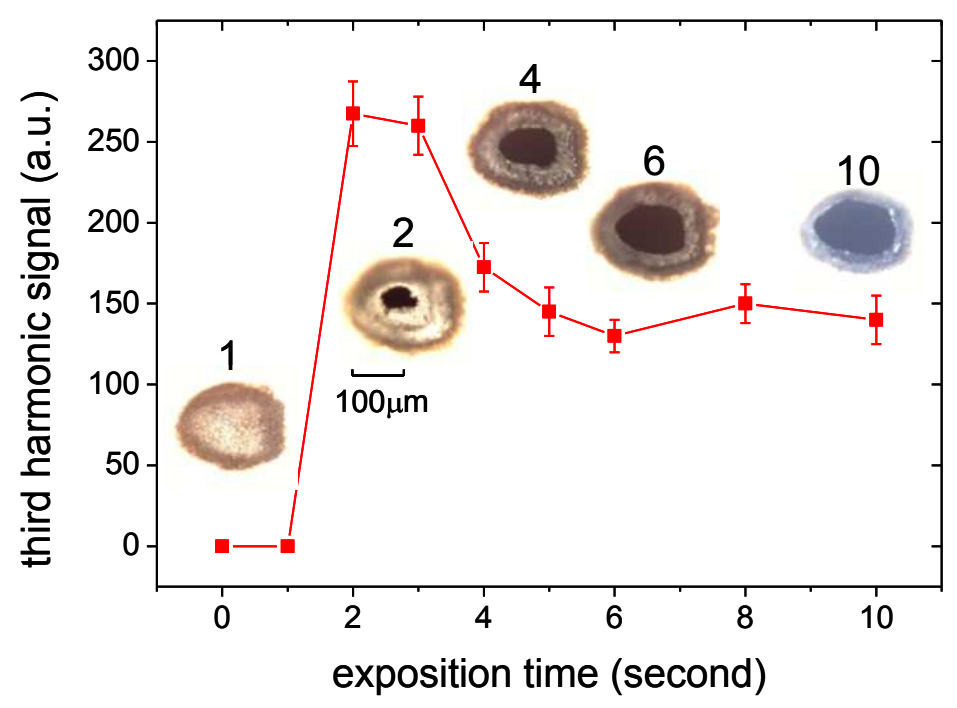

Fig. 7. Third harmonic signal measured after the insert of a $150 \mu \mathrm{m}$ thickness $\mathrm{Al}$ foil around the center of a $4 \mathrm{~cm}$ long filament. The inset present the photos of the filament drilled pinholes, with the numbers on their top indicating the exposition time. 


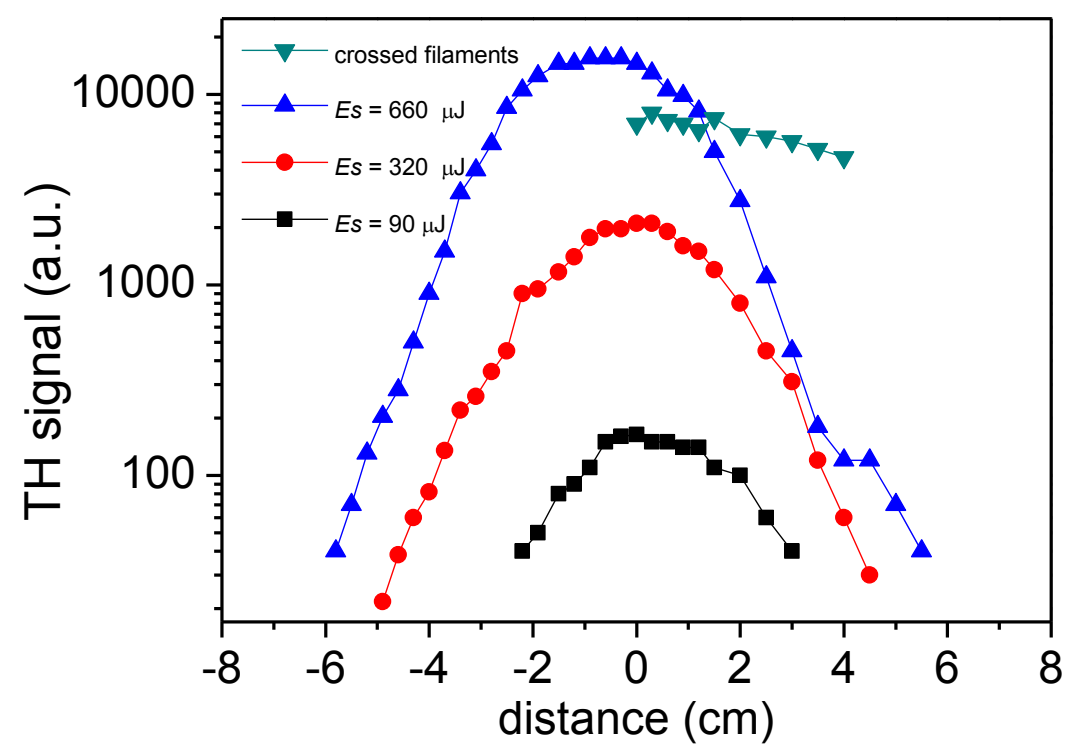

Fig. 8. (a) Third harmonic energy after the pinhole created by the filament core on the aluminum foil. The results from a single filament are presented for three different incident energies. For crossed filaments, a pre-formed $100 \mu \mathrm{m}$ pinhole is scanned after the filament cross at $z=0 \mathrm{~cm}$. The energies of the signal and pump pulse are $320 \mu \mathrm{J}$ and $300 \mu \mathrm{J}$.
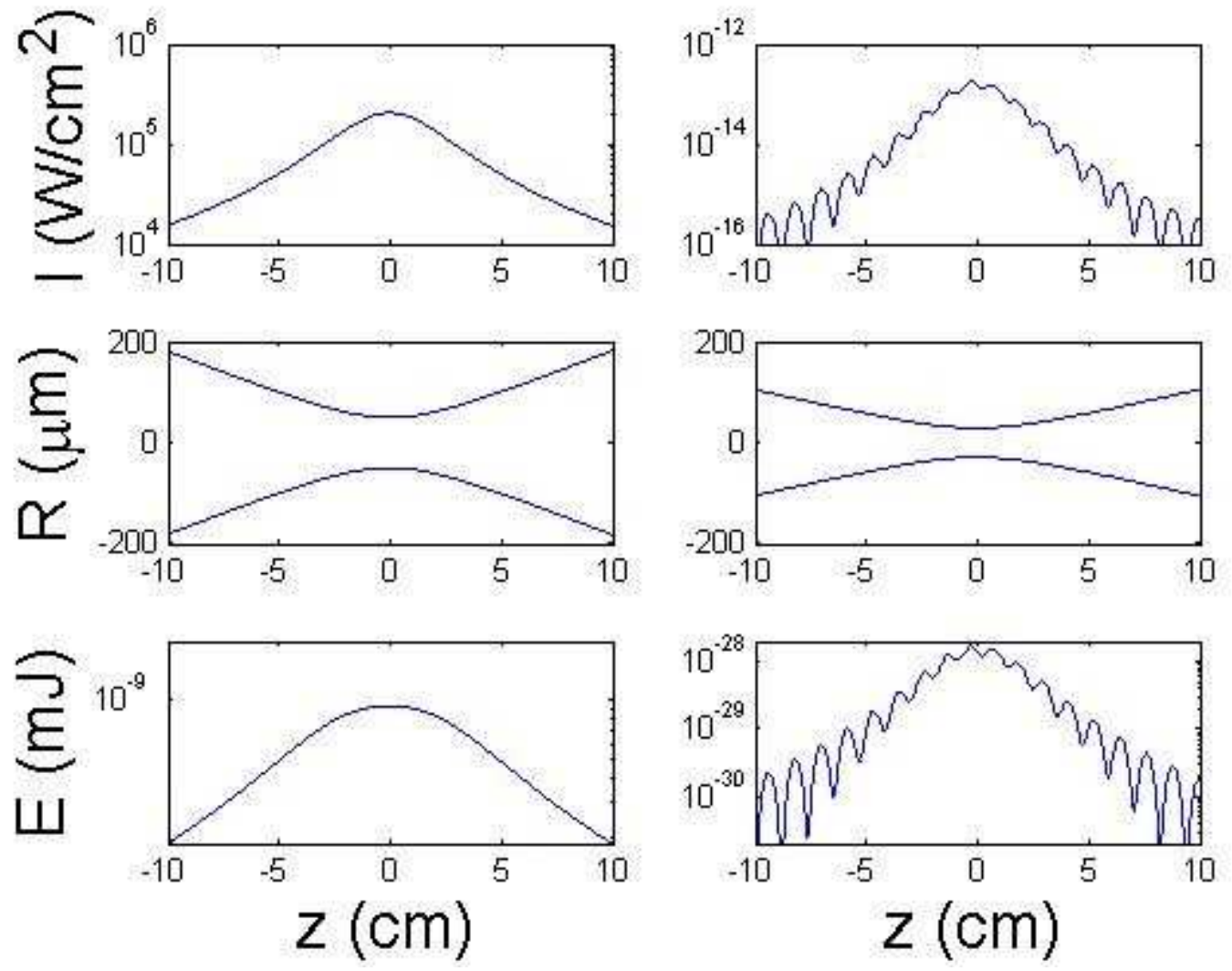
Fig. 9. Propagation dynamics of the fundamental (first column) and the TH (second column) pulses at low energy. First line: Maximum intensity as a function of the propagation distance $z$. Second line: Beam FWHM vs $z$, calculated from the fluence distributions of the fundamental and third harmonic, respectively. Third line: Energy contained within a cylinder of radius 100 microns. The energy of the fundamental pulse is $1 \mathrm{pJ}$ and its waist at focus $z=0 \mathrm{~cm}$ is 100 microns.
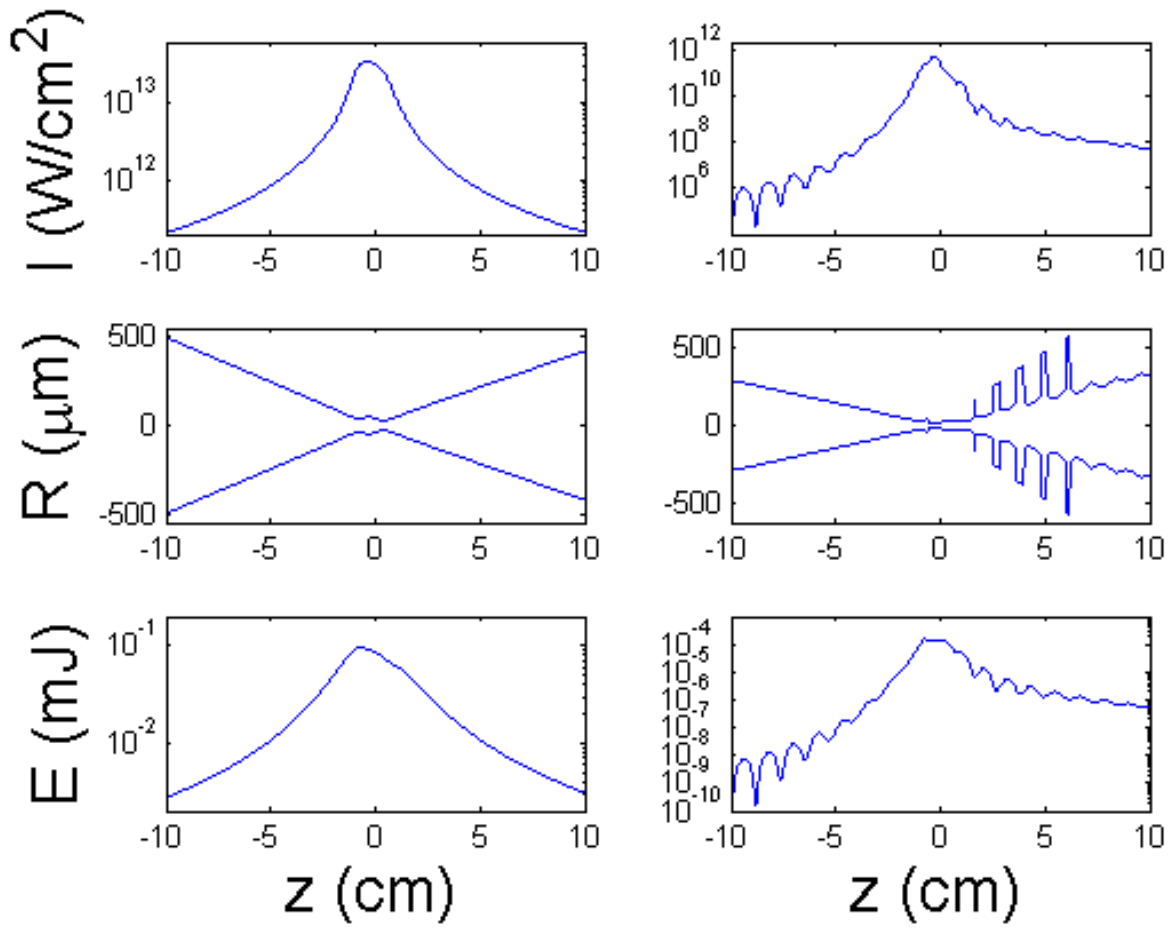

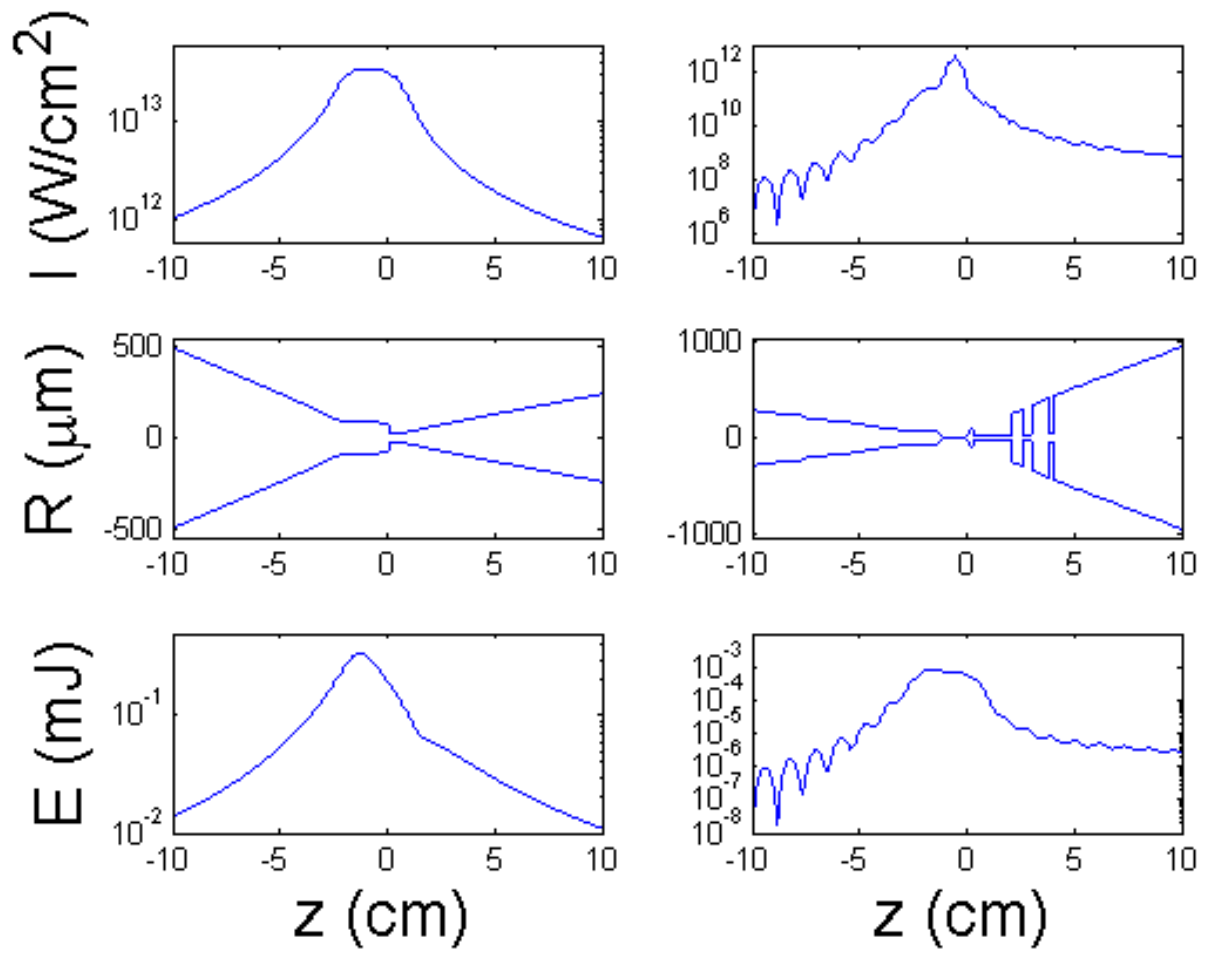

Fig. 10. Propagation dynamics of the fundamental (first column) and the TH (second column) pulses in the conditions of the experiment. The energy of the fundamental pulse is $100 \mu \mathrm{J}$ (top figure) and $500 \mu \mathrm{J}$ (bottom figure) and in both cases, its waist at focus $z=0 \mathrm{~cm}$ would be 30 microns if the propagation was linear. The same quantities as in Fig. 9 are presented but the log scale was used in the first and third line to stress that the energy in the third harmonic pulse does not entirely return to the pump. 

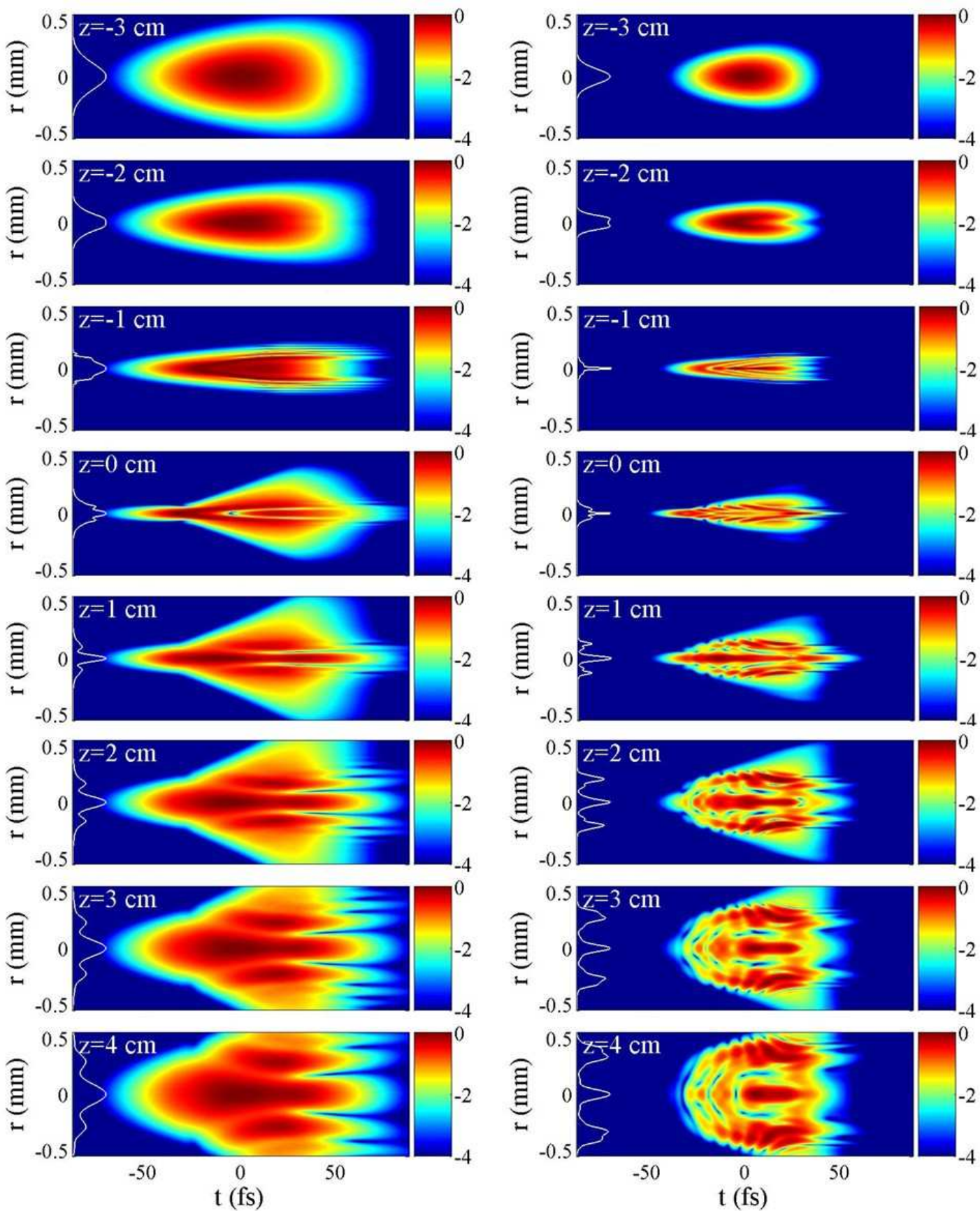

Fig. 11. Spatiotemporal dynamics of the fundamental (left column) and the TH (right) pulses as a function of the propagation distance. The energy of the fundamental pulse is $500 \mu \mathrm{J}$ and its waist at focus $z=0 \mathrm{~cm}$ would be 30 microns if the propagation was linear. The white curves on the left of each subfigure represent the fluence profiles for the fundamental and the TH pulses. 


\section{Reference:}

[1] J. F. Ward, G. H. C. New, Phys. Rev. 185 (1969) 185.

[2] A. L'Huillier, Ph. Balcou, Phys. Rev. Lett. 70 (1993)774.

[3] J. J. Macklin, J. D. Kmetec, C. L. Goudon, Phys. Rev. Lett. 70 (1993)766.

[4] S. Backus, J. Peatross, Z. Zeek, A. Rundquist, G. Taft, M. M. Murnane, H. C. Kapteyn, Opt. Lett. 21 (1996) 665.

[5] A. B. Fedotov, N. I. Koroteev, M. M. T. Loy, X. Xiao, A. M. Zheltikov, Opt. Commun. 133 (1997) 587.

[6] J. Peatross, S. Backus, J. Zhou, M. M. Murnane, H. C. Kapteyn, J. Opt. Soc. Am. B 15(1998) 186.

[7] R. A. Ganeev, M. Suzuki, M. Baba, H. Kuroda, I. A. Kulagin, Appl. Opt. 45 (2006) 748.

[8] D. S. Steingrube, E. Schulz, T. Binhammer, T. Vockerodt, U. Morgner, M. Kovačev, Opt. Express, 17 (2009) 16177.

[9] N. Aközbek, A. Iwasaki, A. Becker, M. Scalora, S. L. Chin, C. M. Bowden, Phys. Rev. Lett. 89 (2002) 143901.

[10] H. Yang, J. Zhang, J. Zhang, L. Z. Zhao, Y. J. Li, H. Teng, Y. T. Li, Z. H. Wang, Z. L. Chen, Z. Y. Wei, J. X. Ma, W. Yu, Z. M. Sheng, Phys. Rev. E 67 (2003) 015401(R).

[11] M. Kolesik, E. M. Wright, A. Becker, J. V. Moloney, Appl. Phys. B 85 (2006) 531.

[12] F. Théberge, N. Aközbek, W. Liu, J. -F. Gravel, S. L. Chin, Opt. Commun. 245 (2005) 399.

[13] N. Kortsalioudakis, M. Tatarakis, N. Vakakis, S. D. Moustaizis, M. Franco, B. Prade, A. Mysyrowicz, A. A. Papadogiannis, A. Couairon, S. Tzortzakis, Appl. Phys. B 80 (2005) 211.

[14] F. Théberge, N. Aközbek, W. Liu, J. Filion, S. L. Chin, Opt. Commun. 276 (2007) 298.

[15] A. Couairon, A. Mysyrowicz, Phys. Rep. 441 (2007) 47.

[16] A. Couairon and A. Mysyrowicz, "Self-focusing : Past and Present Fundamentals and Prospects", Springer Series : Topics in Applied Physics, Vol. 114, R. Boyd, W. Lukishova, G. Svetlana, Y. R. Shen, (Eds.) 2009.

[17] S. Suntsov, D. Abdollahpour, D. G. Papazoglou, S. Tzortzakis, Opt. Express. 17 (2009) 3190.

[18] X. Yang, J. Wu, Y. Peng, Y. Tong, S. Yuan, L. Ding, Z. Xu, H. Zeng, Appl. Phys. Lett. 95 (2009) 111103.

[19] S. Suntsov, D. Abdollahpour, D. G. Papazoglou, S. Tzortzakis, Phys. Rev. A 81 (2010) 033817.

[20] T. T. Xi, X. Lu, and J. Zhang, Opt. Commun. 282 (2009) 3140.

[21] Z. Zhang, X. Lu, Y. Zhang, M. Zhou, T. Xi, Z. Wang, J. Zhang, Opt. Lett. 35 (2010) 974.

[22] R.W. Boyd, "Nonlinear Optics", Academic Press, San Diego, 2008, 3rd ed.

[23] W. Liu, J. -F. Gravel, F. Théberge, A. Becker, S. L. Chin, Appl. Phys. B 80 (2005) 857.

[24] W. Liu, F. Théberge, E. Arévalo, J. -F. Gravel, A. Becker, S. L. Chin, Opt. Lett. 30 (2005) 2602.

[25] T. Brabec, F. Krausz, Phys. Rev. Lett. 78 (1997) 3282.

[26] A. Couairon, E. Gaižauskas, D. Faccio, A. Dubietis, P. Di Trapani, Phys. Rev. E 73 (2006) 016608.

[27] E. R. Peck, K. Reeder, J. Opt. Soc. Am. 62 (1972) 958. 